\title{
Diferencias de género en matemáticas y lengua: rendimiento académico, autoconcepto y expectativas
}

\author{
Carolina Cárcamo ${ }^{\mathrm{ab} *}$, Amparo Moreno ${ }^{\mathrm{a}}$ C Cristina del Barrio ${ }^{\mathrm{a}}$
}

a Universidad Autónoma de Madrid, Madrid, España

${ }^{b}$ Universidad Tecnológica de Bolívar, Cartagena, Colombia

Recibido el 20 de febrero de 2020; aceptado el 22 de abril de 2020

\section{PALABRAS CLAVE}

Estereotipos,

diferencias de género, autoconcepto académico, escuela primaria

\section{KEYWORDS}

Stereotypes, gender differences, academic self-concept, primary school

\begin{abstract}
Resumen Estudios previos muestran diferencias de género en el rendimiento de matemáticas y lengua, principalmente en adolescentes. Este estudio tiene como objetivo evaluar las diferencias de género en autoconcepto, valor subjetivo, expectativas y rendimiento académico; analizar las relaciones entre las variables y conocer el papel que ellas desempeñan en la explicación del rendimiento en lengua y matemáticas. Participaron 406 estudiantes de $4^{\circ}$ y $5^{\circ}$ de primaria en Colombia. Los resultados muestran que las niñas presentan mayores expectativas de rendimiento en matemáticas y lengua, así como un rendimiento en lengua superior a los niños. No se encontraron diferencias de género en el autoconcepto y el valor subjetivo de la tarea. El autoconcepto, las expectativas de rendimiento y la edad, explicaron el rendimiento tanto en matemáticas como en lengua. Se discuten las implicaciones de estos resultados para la disminución de la brecha de género en el aprendizaje. Como este estudio es un primer intento de explorar las relaciones entre las creencias de expectativa y valores de las tareas en matemática y lengua con base en el Modelo de Expectativa-Valor en estudiantes hispanos en primaria, se necesita más investigación para apoyar o refutar estos hallazgos.
\end{abstract}

(c) 2020 Fundación Universitaria Konrad Lorenz. Este es un artículo Open Access bajo la licencia CC BY-NC-ND (http://creativecommons.org/licenses/bync-nd/4.0/).

\section{Gender Differences in Math and Reading: Performance, Self-Concept and Expectations}

Abstract Previous studies show gender differences in math and language performance, mainly in adolescents. In this study, we aim to evaluate gender differences in self-concept, subjective value, expectations and academic performance, to analyze the relationships between the variables, and to understand their role in explaining performance in language and mathematics. A total of 406 th and 5th grade students from Colombia participated in this study. The results show that girls have higher performance expectations in mathematics and language, as well as higher performance in language than boys. No gender differences were found in self-

\footnotetext{
* Autor para correspondencia.

Correo electrónico: carcamo.carolina@hotmail.com
} 
concept and the subjective value of the task. Self-concept, performance expectations and age explained performance in both mathematics and language. We discuss the implications of these results for narrowing the gender gap in learning. Because this study is the first attempt to explore the relationships between expectations and task-value beliefs in language and mathematics in Hispanic primary school students based on the Expectancy-Value Model, more research is needed to support or refute our findings.

(c) 2020 Fundación Universitaria Konrad Lorenz. This is an open access article under the CC BYNC-ND license (http://creativecommons.org/licenses/bync-nd/4.0/).

Las habilidades cognitivas como la capacidad de cálculo y comprensión lectora tienen importantes implicaciones económicas y sociales (Leuven, Oosterbeek, \& Van Ophem, 2004). Específicamente, las diferencias de género en estas habilidades acarrean consecuencias importantes en las decisiones futuras y el bienestar de los/as niños/as al convertirse en factores predictores de sus aspiraciones educativas, laborales (Buser, Niederle \& Oosterbeek, 2014; Gunderson, Ramírez, Levine \& Beilock, 2012; Widlund, Tuominen, Tapola \& Korhonen, 2020) e ingresos salariares (Murnane, Willett, Yves \& Tyler, 2000).

De forma generalizada, las evaluaciones internacionales han documentado diferencias por género en el rendimiento académico en dichas habilidades. Concretamente, en las evaluaciones TIMSS (Trends in International Mathematics y Science Study) y PIRLS (Progress in International Lengua Literacy Study) para estudiantes de $4^{\circ}$ y $5^{\circ}$, las niñas obtienen mejores resultados que los niños en lectura y escritura, pero quedan rezagadas en matemáticas (Meinck \& Brese, 2019; Mullis, Foy \& Hooper 2017).

Asimismo, en la mayoría de los países que participaron en PISA (Programme for International Student Assessment), los adolescentes lograron puntuaciones un poco más altas (5 puntos) en matemáticas que las niñas (OECD, 2018). Mientras que en todos los países las adolescentes obtuvieron puntuaciones significativamente más altas (30 puntos) en habilidad lectora que ellos (OECD, 2018). Sin embargo, en pocos países los adolescentes superan a las chicas en matemáticas. Específicamente, en Colombia la diferencia por género en matemáticas es una de las más grandes (20 puntos), y en lectura la brecha a favor de las chicas es una de las más pequeña (10 puntos) (OECD, 2018).

Dentro de las explicaciones a la brecha académica, algunos estudios apoyan la hipótesis de diferencias biológicas, al afirmar que las niñas tienen habilidades verbales innatas superiores a los niños y estos, a su vez, son superiores en matemáticas (Echavarri, Godoy \& Olaz, 2007; Maccoby \& Jacklin, 1974; Mealey, 2000). Otros estudios, como el presente, apoyan la hipótesis de similitudes de género, y señalan que hombres y mujeres son más similares que diferentes en las habilidades verbales y matemáticas y que, además, las diferencias son principalmente sociales y culturales, constituidas a partir de los roles históricos (Hyde, 2005, 2014).

Entre los factores psicosociales de mayor relevancia se encuentran las creencias que las/los estudiantes tienen de sus habilidades, que se asocian a la motivación y el rendimiento, afectando la elección de las actividades que realizan y el mantenimiento de un comportamiento. Por tanto, cuanto más fuerte sean las creencias de autoeficacia, mayor será el esfuerzo y persistencia para enfrentar obstáculos (Bandura, 1977; Eccles et al., 1983). En esta línea, el Modelo de Expectativa-Valor (Expectancy-Value Model of Achievement Choices) (MEV) establece que tanto las creencias de las/los estudiantes sobre sus competencias en matemáticas y lengua como el autoconcepto, las expectativas y el valor subjetivo de las tareas están relacionados con la motivación y predicen el rendimiento y la elección de actividades futuras (Eccles et al., 1983; Eccles, 2009; Wigfield et al., 1997).

Otra variable que influye en las creencias de autoeficacia y afecta las decisiones de niños/as, incluso antes de que existan diferencias en rendimiento, son los estereotipos. Estos determinan, en buena medida, aspectos cruciales para la vida de niños y niñas como la motivación para realizar diferentes tareas (Eccles et al., 1983; Hyde, 2005, 2014). Por ejemplo, a los cinco años, niños/as asocian la inteligencia con su propio género, pero a partir de los seis años las niñas son menos propensas que los niños a creer que las mujeres son inteligentes, y suelen tener un menor interés en participar en actividades etiquetadas para niños muy inteligentes (Bian, Leslie \& Cimpian, 2017).

Específicamente, existe una tendencia estereotipada a ver las matemáticas como un dominio masculino y la lectura como un dominio femenino (Brandell \& Staberg, 2008; Eccles et al., 1983; Steele, 1997). En consecuencia, los niños entre seis y diez años han adquirido este estereotipo y consideran de forma implícita y explícita que las matemáticas son más para ellos que para las niñas, quienes se identifican de forma más débil con las matemáticas (Cvencek, , Meltzoff \& Greenwald, 2011). Curiosamente, incluso si niñas y niños reciben calificaciones similares en matemáticas, las niñas se perciben como menos hábiles que los niños en este dominio (Frenzel, Pekrun \& Goetz, 2007).

Igualmente, en la adolescencia las mujeres presentan resultados inferiores en las pruebas de matemáticas cuando son conscientes de un estereotipo negativo sobre sus habilidades que cuando lo desconocen (Spencer, Steele \& Quinn, 1999). Específicamente, las adolescentes tienen un autoconcepto inferior de sus habilidades en matemáticas, aunque los resultados reales no difieran entre ellos y ellas (Sainz \& Eccles, 2012), así como expectativas de rendimiento más bajas que los hombres en matemáticas (Eccles et al., 1983; Gunderson et al., 2012).

Por todo lo anterior, no es de extrañar que las adolescentes se sientan menos seguras y desarrollen un mayor interés hacia las ciencias biológicas y sociales, evitando cursos avanzados y carreras profesionales en ciencia, tecnología e ingeniería (Buser et al., 2014; Eccles et al., 1983; Goldin, Katz \& Kuziemko, 2006; Gunderson et al., 2012; Sainz \& Eccles, 2012), y que los adolescentes muestren mayor interés hacia la física y la ingeniería (Eccles, Terry \& Judith, 
1984; OECD, 2016). Además, el interés de las/los estudiantes en matemáticas o lenguaje está relacionado con el valor que dan a una tarea (Wigfield et al., 2015).

El MEV es uno de los aportes más importantes a la comprensión de la motivación de logro, siendo una fuente significativa de investigación para explicar las diferencias entre niños y niñas en las últimas décadas. Sin embargo, los estudios se han centrado en el alumnado de secundaria y educación superior (Eccles et al., 1983; Gunderson et al., 2012; Guo, Marsh, Morin, Parker \& Kaur et al., 2015) y, en menor medida, al de educación primaria (Jacobs, Lanza, Osgood, Eccles \& Wigfield 2002), cuando se van formando habilidades cognitivas y sociales responsables del desarrollo de autoconceptos y expectativas académicas (Harter, 2012).

Pese a la influencia del contexto cultural en las variables incluidas en el MEV, la mayoría de los estudios se han realizado en Estados Unidos, mientras que muy pocos en países europeos (Sainz \& Eccles, 2012; Sainz \& Upadyaya, 2016). A su vez, no se disponen investigaciones empíricas publicadas en países latinoamericanos con este modelo; por tanto, se desconoce qué tan generalizable son estos resultados. Por ende, existe una necesidad reconocida de más estudios que prueben el MEV en diferentes niveles educativos y contextos (Pinxten et al., 2015; Sainz \& Eccles, 2012).

De acuerdo con ello, esta investigación se plantea, como objetivo general, contribuir a la comprensión del rendimiento académico en las áreas de lengua y matemáticas en los/as niños/as colombianos/as en el marco del MEV. Específicamente, el primer objetivo es analizar las posibles diferencias de género en el autoconcepto con respecto a los dominios de conocimiento, el valor subjetivo de la tarea, las expectativas y el rendimiento en niños/niñas. El segundo objetivo es examinar las posibles asociaciones entre estas variables. El tercer objetivo es contrastar el papel que puede desempeñar el género en la explicación del rendimiento académico. Respecto al primer y segundo objetivo, y teniendo en cuenta los resultados de investigaciones previas, se hipotetiza la existencia de diferencias significativas en el autoconcepto, el valor de la tarea, las expectativas y el rendimiento a favor de las niñas en lengua, y de los niños en matemáticas (hipótesis 1). Asimismo, se espera encontrar asociaciones positivas entre las variables (hipótesis 2). En relación con el último objetivo, se plantea que el género, el autoconcepto y las expectativas de éxito serán las variables más influyentes en la explicación del rendimiento académico en lengua y matemáticas (hipótesis 3 ).

\section{Método}

\section{Participantes}

Se seleccionaron 406 estudiantes (58.9\% niñas) de estrato socioeconómico medio, entre ocho y once años $(M=9.52 ; D E=.75)$. De ellos, $196(48.3 \%)$ cursaban $4^{\circ} \mathrm{y}$ $210(51.7 \%) 5^{\circ}$ de primaria en tres escuelas públicas de Cartagena, Colombia. Dadas las características generales del estudio para la selección de los participantes, se optó por un muestreo no probabilístico de conveniencia.

\section{Variables e instrumentos}

Los/as estudiantes respondieron a un cuestionario con dos secciones. La primera, contenía preguntas para obtener información sociodemográfica y en la segunda sección las escalas descritas a continuación.

Autoconcepto de habilidad en matemáticas y lengua. Se refiere a la percepción de habilidad propia en cada dominio. Esta escala se basó en la escala del MEV para lengua y matemáticas (Eccles \& Harold, 1991). Este cuestionario incluye cinco preguntas sobre el concepto que tienen los/as niños/as de sus habilidades en cada dominio; por ejemplo: “QQué tan bueno eres en matemáticas?”, o "¿Qué tan bien esperas que te vaya en castellano? [término utilizado en Colombia para la asignatura de Lengua]. Las posibles respuestas van en un continuo del 1 al 7 , donde 1 es "nada bueno" y 7 es "muy bueno". Se obtiene una puntuación total en esta escala promediando los valores obtenidos en todos los ítems. La consistencia interna de estas escalas fue de .81 y .76 , respectivamente. La prueba de esfericidad de Bartlett es estadísticamente significativa tanto para matemáticas como para lengua $\left[X^{2}(10)=671.928\right.$, $p=.000 ; X^{2}(10)=434.305, p=.000$, respectivamente]. La medida de Kaiser de la adecuación del muestreo fue de .82 en matemáticas y .81 en lengua. Además, el primer factor explica el $48.77 \%$ de la varianza, mientras que el de lengua explica el $39.53 \%$ de la varianza total. En países de habla hispana, las escalas similares han demostrado adecuadas propiedades psicométricas (Sainz \& Eccles, 2012).

Valor de matemáticas y lengua. Se refiere a la percepción de la utilidad, el interés y el valor de cada dominio para cada participante. Esta escala se sustenta en el MEV. La componen cinco preguntas con una redacción semejante sobre la importancia que tienen estos dominios para los/as niños/as; por ejemplo: “¿Qué tan útil es lo que aprendes en matemáticas?”, o “¿Cuánto te gusta dedicarte al castellano?”. La puntuación de las respuestas es de 1 a 7 y se promedia. Tras los análisis, se encuentra un índice de confiabilidad de .62 y .70 para matemáticas y lengua, respectivamente. La prueba de esfericidad de Bartlett es estadísticamente significativa para matemáticas y lengua $\left[X^{2}(10)=242.452\right.$, $p=.000 ; X^{2}(10)=443.788, p=.001$, respectivamente]. La medida de Kaiser de la adecuación del muestreo fue de .68 en matemáticas y .69 en lengua. Además, el primer factor explica el $26.63 \%$ de la varianza, mientras que el de lengua explica el $29.46 \%$ de la varianza.

Expectativa de rendimiento en matemáticas y lengua. Esta escala consistió en un elemento con cinco opciones donde las/los estudiantes informaron de la calificación que creían que obtendrían en las asignaturas al final del año académico. Las respuestas oscilan entre 1 (fallido) a 5 (excelente) correspondientes al sistema de calificación colombiano.

Rendimiento en matemáticas y lengua. Consiste en la nota final obtenida promediando las evaluaciones del año escolar de las actividades realizadas por las/los estudiantes en ambos dominios reportada por las escuelas.

\section{Procedimiento}

Se contactaron a las/los coordinadores/as de las escuelas para presentar el proyecto y se obtuvo el permiso para dirigirse a todas las familias e informarles el objetivo del estudio y solicitar el consentimiento para la participación de sus hijos/as. Puesto que se incluyó una muestra más joven que las utilizadas en estudios previos en población de habla hispana, se tuvo cuidado para asegurar la comprensión de 
las preguntas. Por ello, el instrumento fue piloteado con 20 estudiantes para verificar la comprensión, realizando pequeñas modificaciones en la redacción de algunos ítems. A los/as niños/as se les explicó que su contribución era voluntaria y se les permitió terminar su participación en cualquier momento. La aplicación de los instrumentos se realizó por cursos, en las aulas y en el horario suministrado por las escuelas. Cada sesión duró entre 30 y 50 minutos.

\section{Análisis de datos}

Además de los indicadores de tendencia central y dispersión, se realizaron análisis de varianza para comparar las medias y analizar las diferencias entre niños y niñas (prueba $t$ de Student para muestras independientes) y se calculó el índice $d$ de Cohen para valorar el tamaño del efecto de las diferencias encontradas. También se calcularon los coeficientes de correlación de Pearson para establecer las asociaciones entre variables por género. Por último, se realizó una regresión lineal múltiple con el objetivo de conocer el porcentaje de varianza de cada dominio, explicado por las distintas variables del estudio; estas se ingresaron en la ecuación como un bloque, ya que no había ninguna razón para ordenarlas. Para analizar los datos se utilizó el paquete estadístico SPSS.

\section{Resultados}

\section{Diferencias por género}

Las medias y las desviaciones estándar de todas las variables del estudio, para la muestra total y por género, se presentan en la tabla 1. Los resultados muestran que los/las estudiantes en promedio presentan altas expectativas de rendimiento, tienen un alto autoconcepto y consideran importantes ambos dominios. Sin embargo, los niños tuvieron una mayor variabilidad que las niñas en el autoconcepto, rendimiento y expectativas de rendimiento en matemáticas y las niñas en el autoconcepto de habilidad en lengua. Los docentes calificaron el rendimiento académico de matemáticas y en lengua de las/los estudiantes en la media.

En concordancia, la prueba $t$ de Student arroja una diferencia a favor de las niñas (véase tabla 1). Específicamente, las niñas presentan mayores expectativas de rendimiento tanto en matemáticas $(t=-3.19 ; g l=277 ; p=.002 ; d=-.32)$ como en lengua $(t=2.08 ; g l=319 ; p=.038 ; d=.10)$, así como un rendimiento en lengua significativamente superior al de los niños $(t=-3.45 ; g l=400 ; p=.001 ; d=-.34)$.

\section{Correlaciones entre variables}

En la tabla 2 se presentan los valores y la relevancia estadística de las correlaciones. Las correlaciones entre el rendimiento y el autoconcepto fueron positivas y significativas entre niñas y niños para matemáticas $\left(r=.43^{* *} ; r=46^{* *}\right.$, respectivamente) y lengua $\left(r=.18^{* *} ; r=.09^{* *}\right)$, así como el rendimiento y las expectativas en matemáticas $\left(r=.26^{* *}\right.$; $\left.r=.21^{* *}\right)$, pero solo en lengua para las niñas $\left(r=.23^{* *}\right.$; $r=.113$ ). El autoconcepto y las expectativas también correlacionaron de forma positiva para niñas y niños en matemáticas $\left(r=.31^{* *} ; r=.31^{* *}\right)$ y lengua $\left(r=.18^{* *} ; r=.23^{* *}\right)$. El valor de la tarea correlacionó de forma significativa con el rendimiento solo para las niñas, de forma positiva para matemáticas $\left(r=.21^{* *}\right)$ y de forma negativa en lengua $\left(r=-.17^{* *}\right)$. El rendimiento en ambas disciplinas correlacionó de forma positiva y significativa para niñas y niños $\left(r=.64^{* *} ; r=.71^{* *}\right)$.

\section{Predictores del rendimiento académico en mate- máticas y lengua}

Rendimiento en matemáticas. El modelo obtenido explicó el $22 \%\left(F_{398}=19.30 ; p=.000\right)$ de la varianza del rendimiento en matemáticas. Como puede observarse en los

Tabla 1 Estadísticas descriptivas y diferencias de género

\begin{tabular}{lcccccccccccc}
\hline \multirow{2}{*}{ Variables } & \multicolumn{3}{c}{ Participantes } & \multicolumn{2}{c}{ Niño } & \multicolumn{1}{c}{ Niña } & \multicolumn{4}{c}{ Diferencia de género } \\
\cline { 2 - 11 } & M & $D E$ & $M$ & $D E$ & $M$ & $D E$ & $t$ & $g l$ & $p$ & $d$ & VR \\
\hline Autoconcepto HM & 5.32 & 1.14 & 5.34 & 1.18 & 5.30 & 1.12 & .40 & 404 & .684 & .03 & 1.05 \\
Valor TM & 5.94 & .85 & 5.99 & .82 & 5.90 & .88 & .94 & 404 & .346 & .05 & .93 \\
Expectativa RM & 4.66 & .55 & 4.59 & .59 & 4.71 & .51 & -2.08 & 319 & .038 & .10 & 1,15 \\
Rendimiento M & 3.6 & .54 & 3.61 & .55 & 3.65 & .52 & -.68 & 400 & .500 & -.07 & 1.05 \\
Autoconcepto HL & 5.83 & .95 & 5.83 & .87 & 5.83 & 1.00 & .08 & 404 & .936 & 0 & .87 \\
Valor TL & 5.92 & .89 & 5.92 & .86 & 5.92 & .91 & -.05 & 404 & .958 & 0 & .94 \\
Expectativa RL & 4.6 & .54 & 4.58 & .63 & 4.76 & .46 & -3.19 & 277 & .002 & -.32 & 1.36 \\
Rendimiento L & 3.68 & .50 & 3.58 & .48 & 3.75 & .50 & -3.45 & 400 & .001 & -.34 & .96 \\
\hline
\end{tabular}

Nota. Las puntuaciones de las escalas van de 1 a 7 (variables 1, 2, 5 y 6). Las puntuaciones de las escalas van de 1 a 5 (variables $3,4,7$ y 8 ). Participaron 239 niños y 167 niñas. $M=$ media. $D E=$ desviación estándar. $d=\mathrm{d}$ de Cohen. $V R=D E$ de niños dividido por $D E$ de niñas. 
Tabla 2 Correlaciones de las variables

\begin{tabular}{lcccccccc}
\hline \multicolumn{1}{c}{ Escalas } & 1 & 2 & 3 & 4 & 5 & 6 & 7 & 8 \\
\hline Autoconcepto HM (1) & - & $.54^{* *}$ & $.31^{* *}$ & $.43^{* *}$ & $.29^{* *}$ & $.13^{*}$ & .07 & $.18^{* *}$ \\
Valor TM (2) & $.48^{* *}$ & - & $.19^{* *}$ & $.21^{* *}$ & $.19^{* *}$ & -.02 & -.02 & .07 \\
Expectativa RM (3) & $.31^{* *}$ & $.34^{* *}$ & - & $.26^{* *}$ & $.20^{* *}$ & $.45^{* *}$ & $.45^{* *}$ & $.16^{*}$ \\
Rendimiento M (4) & $.46^{* *}$ & .13 & $.21^{* *}$ & - & $.18^{* *}$ & -.05 & $.21^{* *}$ & $.64^{* *}$ \\
Autoconcepto HL (5) & $.33^{* *}$ & $.26^{* *}$ & $.27^{* *}$ & .07 & - & $.52^{* *}$ & $.18^{* *}$ & $.18^{* *}$ \\
Valor TL (6) & .14 & $.43^{* *}$ & $.20^{* *}$ & -.08 & $.52^{* *}$ & - & $20^{* *}$ & $-.17^{* *}$ \\
Expectativa RL (7) & -.06 & .12 & $.40^{* *}$ & .10 & $.23^{* *}$ & $.27^{* *}$ & - & $.23^{* *}$ \\
Rendimiento L (8) & $.35^{* *}$ & .07 & $.21^{* *}$ & $.71^{* *}$ & .09 & -.15 & .11 & - \\
\hline
\end{tabular}

Nota. El resultado de las niñas se muestra en la diagonal superior y el de los niños en la diagonal inferior.

${ }^{*} p \leq .05,{ }^{* *} p \leq .01$.

resultados de la regresión que aparecen en la tabla 3, la edad $(\beta=-.16 ; p=.005)$ y el curso académico $(\beta=-.17$; $p=.040$ ) se relacionaron negativamente con el rendimiento, mientras que el autoconcepto $(\beta=.43 ; p=.000)$ y las expectativas de rendimiento $(\beta=.13 ; p=.006)$ lo explicaron de manera positiva. Es decir, los/las estudiantes con menor edad y curso académico, un autoconcepto superior y altas expectativas presentaron un rendimiento académico en matemáticas superior que aquellos con mayor edad, curso académico superior, bajo autoconcepto y expectativas inferiores de rendimiento.

Rendimiento en lengua. El modelo obtenido explicó el $16 \%\left(F_{395}=12.97 ; p=0.00\right)$ de la varianza del rendimiento en lengua. El género $(\beta=.13 ; p=.005)$, el autoconcepto $(\beta=.28 ; p=.000)$ y las expectativas de rendimiento $(\beta=.16 ; p=.001)$ mostraron una relación positiva con el rendimiento en lengua, mientras que la edad $(\beta=-.14$; $p=.022)$ y el valor de la tarea $(\beta=-.32 ; p=.000)$ una relación negativa. Por tanto, las niñas, estudiantes con menor edad, autoconcepto y expectativas de rendimiento superiores presentaron mayor rendimiento académico en lengua que los niños, estudiantes con mayor edad, niveles inferiores de autoconcepto y expectativas de éxito (véase tabla 3).

\section{Discusión y conclusiones}

\section{Diferencias por género}

Como primer objetivo de este estudio, se analizaron posibles diferencias de género en autoconcepto, valor de la tarea, expectativas de éxito y rendimiento académico en niños/as de primaria. Los resultados obtenidos apoyan parcialmente la primera hipótesis planteada, puesto que se corroboran diferencias de género en las expectativas para ambos dominios y el rendimiento en lengua, pero no se encuentran diferencias significativas en el autoconcepto y el valor subjetivo de la tarea.

Por tanto, se concluye que a temprana edad las niñas muestran expectativas más positivas de su rendimiento en lengua y matemáticas. Este hallazgo es consistente con estudios que han mostrado que las niñas presentan creencias

Tabla 3 Regresión entre las variables asociadas al rendimiento en matemáticas y lengua

\begin{tabular}{lcccccccc}
\hline & \multicolumn{3}{c}{ Variables } & \multicolumn{3}{c}{ Rendimiento en matemáticas } & \multicolumn{4}{c}{ Rendimiento en lengua } \\
\cline { 2 - 9 } & $\mathrm{B}$ & $\mathrm{EE}$ & $\mathrm{B}$ & $\mathrm{p}$ & $\mathrm{B}$ & $\mathrm{EE}$ & $\mathrm{B}$ & $\boldsymbol{p}$ \\
\hline Constante & 2.97 & .38 & & .000 & 3.86 & .36 & .000 \\
Género & .01 & .04 & .01 & .791 & .13 & .04 & .13 & .005 \\
Edad & -.11 & .04 & -.16 & .005 & -.92 & .04 & -.14 & .022 \\
Curso & -.13 & .06 & -.17 & .040 & -.00 & .06 & -.00 & .931 \\
Autoconcepto & .20 & .02 & .43 & .000 & .14 & .02 & .28 & .000 \\
Valor T & -.03 & .03 & -.06 & .251 & -.17 & .03 & -.32 & .000 \\
Expectativa R & .12 & .04 & .13 & .006 & .15 & .04 & .16 & .001 \\
R2 & 2.97 & & & & .22 & & & \\
Adj R2 & .013 & & & & .21 & & & \\
\hline
\end{tabular}

Nota. $\mathrm{EE}=$ error estándar. 
más positivas en su rendimiento en lengua (Jacobs et al., 2002); por el contrario, difiere de investigaciones que reportan expectativas superiores en matemáticas en niños (Cvencek et al., 2011; Jacobs et al., 2002). Sin embargo, otras pesquisas han encontrado que las niñas a los cuatro años creen que son académicamente superiores a los niños, quienes asumen este estereotipo a partir de los siete años (Hartley \& Sutton, 2013). Igualmente, niños y niñas piensan desde los cinco años que las niñas disfrutan más de las matemáticas que los niños (Paz-Albo, Cvencek, Herranz-Llácer, Hervás-Escobar \& Meltzoff 2017). Consecuentemente, en edades más tempranas, las niñas pueden presentar creencias estereotipadas en lengua y no participan del estereotipo que las desfavorece en matemáticas, donde ellas esperan desempeñarse mejor.

Si bien en las pruebas internacionales, los niños tienen un rendimiento superior en matemáticas y las niñas en lengua (Mullis et al., 2017), en este estudio las niñas presentan un rendimiento en lengua significativamente superior al de los niños, pero no se encuentran diferencias en el rendimiento en matemáticas. Es decir, las niñas colombianas que participaron en este estudio son, en promedio, igual de buenas en matemáticas que los niños, pero los superan en lengua. Existen algunos factores que favorecen estos resultados académicos. Por ejemplo, las niñas pasan más tiempo haciendo los deberes escolares y leyendo por diversión, mientras que los niños emplean más tiempo en videojuegos (OECD, 2015).

La ausencia de diferencias en matemáticas es coincidente con la reducción de la brecha a nivel internacional, tal como se ha dado en Colombia desde el 2015, debido a una continua mejoría en el desempeño de las niñas (OECD, 2018), situación que se podría reflejar en las notas escolares.

\section{Asociación entre variables por género}

Como segundo objetivo, se examinaron las posibles asociaciones entre las variables y los resultados ratificaron la segunda hipótesis. Ciertamente, se encuentran en su mayoría correlaciones positivas y significativas entre el rendimiento, el autoconcepto y las expectativas para los/as estudiantes en matemáticas. Este patrón de correlaciones concuerda con el MEV, donde se estipula que el autoconcepto de habilidad influye en las expectativas de éxito y el valor de la tarea, variables que a su vez afectan el rendimiento y la elección de la tarea (Eccles et al., 1983). Resultados equivalentes se han encontrado en los/as adolescentes con correlaciones significativas entre el rendimiento y el autoconcepto de habilidad matemática (Sainz \& Eccles, 2012).

No obstante, en el caso de la lengua estas variables solo son significativas para las niñas, mientras que para los niños no son significativas y solo correlacionan con las variables del dominio no coincidente. Estos resultados pueden interpretarse a la luz del Modelo de Comparación Interna/ Externa (MCl/E) y la Teoría de Comparación Dimensional. El $\mathrm{MCl} / \mathrm{E}$ postula que los/as estudiantes forman sus autoconceptos al compararse con las actuaciones de sus pares en una disciplina y contrastan sus propios logros en otros dominios no coincidentes. Mientras que la comparación dimensional plantea que el rendimiento tiene efectos positivos en el autoconcepto del dominio coincidente, pero efectos negativos en el dominio del autoconcepto no coincidente (Guo, Marsh, Parker \& Dicke, 2018; Marsh \& Hau, 2004).
Investigaciones en primaria han encontrado que el rendimiento en lengua correlaciona de forma positiva y significativa con el rendimiento en matemáticas, mientras que los autoconceptos de lengua y matemáticas tienden a no estar correlacionados (Kavanagh, 2019; Marsh et al., 2018). Por tanto, para los niños tener altas calificaciones en lengua podría no hacerlos concluir que son talentosos de forma verbal, pero tener alta puntuación en matemáticas sí podría llevarlos a considerar que son y serán talentosos en lengua. El hecho de que estas asociaciones sean solo significativas para las niñas, también puede reflejar la diferencia de género en el significado asociado y atribuciones causales con las habilidades en lengua.

\section{Factores determinantes del rendimiento académico en matemáticas y lengua}

El autoconcepto académico y las expectativas fueron, en ese orden, las variables con una mayor asociación positiva en la explicación del rendimiento en ambos dominios. Este resultado coincide con un reciente estudio en el contexto latinoamericano, donde las creencias de autoeficacia de las/los adolescentes acerca de sus habilidades en matemáticas, predijo un mejor rendimiento matemático y creencias de igualdad de género (Smith-Castro, Montero-Rojas, Moreira-Mora \& Zamora-Araya, 2019). Asimismo, investigaciones han mostrado la importancia de que los y las estudiantes desarrollen expectativas de éxito y un autoconcepto positivo, para favorecer su rendimiento escolar, aspiraciones y ayudarles a enfrentar los cambios (Guo et al., 2015; Guo et al., 2018; Marsh et al., 2018; Wigfield \& Gladstone, 2019).

El valor de la tarea solo explicó de forma negativa el rendimiento en lengua, pero no en matemáticas. Es decir, cuanto mayor es la importancia de la tarea, menor es el rendimiento en lengua. No obstante, se ha encontrado que esta variable está más relacionada con la elección de tareas que con el rendimiento (Wigfield \& Eccles, 2000). Sin embargo, el valor de la tarea puede predecir de forma positiva la comprensión lectora (Anmarkrud \& Bråten, 2009). Posiblemente, la relación negativa entre las variables estudiadas puede estar influida por el objetivo y la forma como se evalúa la asignatura; por tanto, los/as participantes pueden asociar la importancia de la lengua más con el estrés de las evaluaciones que con el valor que se le conceda a la asignatura.

En cuanto a las variables sociodemográficas, a medida que los/as niños/as del estudio aumentan en edad, el rendimiento en ambos dominios se deteriora, así como al aumentar el curso, pero solo para el rendimiento en matemáticas. El género solo aportó a la explicación del rendimiento en lengua, pero no en matemáticas. Estos resultados concuerdan con la reducción de las diferencias en matemáticas a nivel internacional (OECD, 2016, 2018). Específicamente, la desigualdad en matemáticas desaparece en algunos países que presentan mayor equidad de género, como Noruega y Suecia, donde las niñas tienen igual rendimiento en matemáticas que los niños, pero se mantienen las diferencias en lengua, superando a los niños (Guiso, Monte, Sapienza \& Zingales, 2008; Stoet \& Geary, 2018).

Teniendo en cuenta los resultados, se resalta la importancia de diseñar programas de intervención que trabajen en las expectativas y el autoconcepto académico de los/as 
niñas/os en ambos dominios. En el caso de la lengua, es necesario poner en marcha acciones dirigidas a la intervención de estereotipos de género de los/las estudiantes y fortalecer en los niños expectativas positivas, así como su desempeño en lengua. Respecto a las matemáticas, las acciones pueden ir orientadas a la prevención de creencias estereotipadas.

El estudio presenta una serie de limitaciones. Primero, las posibles restricciones de la validez externa inherentes al muestreo no probabilístico y el diseño transversal. Segundo, el uso de un solo indicador para medir una variable, por ejemplo, las notas académicas, no manifiestan todos los conocimientos incluidos en el currículo. Del mismo modo, la confiabilidad media baja de algunas de las medidas (Castañeda, Cabrera, Navarro \& Vries 2010) podrían mejorarse, no obstante, estas garantizan suficientemente la bondad de las escalas. Tercero, los resultados muestran diferencias entre los niños y las niñas en las expectativas en ambos dominios y el rendimiento en lengua, pero se desconocen las atribuciones causales (Weiner, 1990), la motivación externa (Rossi, Trevisol, dos Santos-Nunes, DepievePatias \& Von Hohendorff, 2020) la regulación emocional (Andrés et al., 2017) y la procrastinación (Domínguez Lara, Prada Chapoñan \& Moreta Herrera, 2019), factores que podrían resultar relevantes para explicar el éxito académico. Nuevas investigaciones podrían contribuir a dar explicación a estos interrogantes mediante la implementación de metodologías mixtas y estudios longitudinales.

\section{Agradecimientos}

Agradecemos a los docentes, las estudiantes y las familias por su participación en este proyecto. También a la Dra. Gloria Berdugo Oviedo, por su revisión de una versión anterior del manuscrito. Esta investigación cuenta con financiación del programa "Bolívar Gana con Ciencia” de la Gobernación de Bolívar (Colombia) y la Fundación Centro de Estudios Interdisciplinarios Básicos y Aplicados (CEIBA), concedida a la primera autora para cursar sus estudios doctorales. Agradecemos el apoyo de la Universidad Tecnológica de Bolívar.

\section{Referencias}

Andrés, M. L., Stelzer, F., Vernucci, S., Canet Juric, L., Galli, J. I., \& Navarro Guzmán, J. I. (2017). Regulación emocional y habilidades académicas: Relación en niños de 9 a 11 años de edad. Suma Psicológica, 24(2), 79-86. https://doi.org/10.1016/j.sumpsi.2017.07.001

Anmarkrud, Ø., \& Bråten, I. (2009). Motivation for reading comprehension. Learning and Individual Differences, 19(2), 252-256. https://doi.org/10.1016/j.lindif.2008.09.002

Bandura, A. (1977). Self-efficacy: Toward a unifying theory of behavioral change. Psychological Review, 84(2), 191-215. https://doi. org/10.1037/0033-295X.84.2.191

Bian, L., Leslie, S., \& Cimpian, A. (2017). Gender stereotypes about intellectual ability emerge early and influence children's interests. Science, 355(6323), 389-391. https://doi.org/10.1126/ science.aah6524

Brandell, G., \& Staberg, E. (2008). Mathematics: A female, male or gender-neutral domain? A study of attitudes among students at secondary level. Gender and Education, 20(5), 495-509. https:// doi.org/10.1080/09540250701805771
Buser, T., Niederle, M., \& Oosterbeek, H. (2014). Gender, competitiveness, and career choices. The Quarterly Journal of Economics, 129(3), 1409-1447. https://doi.org/10.1093/qje/qju009

Castañeda, M., Cabrera, A., Navarro, Y., \& Vries, W. (2010). Procesamiento de datos y análisis estadísticos utilizando SPSS: un libro práctico para investigadores y administradores educativos. EDIPUCRS.

Cvencek, D., Meltzoff, A. N., \& Greenwald, A. G. (2011). MathGender stereotypes in elementary school children. Child Development, 82(3), 766-779. https://doi.org/10.1111/j.14678624.2010.01529.x

Domínguez Lara, S., Prada Chapoñan, R., \& Moreta Herrera, R. (2019). Diferencias de género en la influencia de la personalidad sobre la procrastinación académica en estudiantes universitarios peruanos. Acta Colombiana de Psicología, 22(2),125-147. https://doi.org/10.14718/ACP.2019.22.2.7

Eccles, J. (2009). Who am I and what am I going to do with my life? Personal and collective identities as motivators of action. Educational Psychologist, 44(2), 78-89. https://doi. org $/ 10.1080 / 00461520902832368$

Eccles, J., \& Harold, R. D. (1991). Gender differences in sport involvement: Applying the eccles' expectancy-value model. Journal of Applied Sport Psychology, 3(1), 7-35. https://doi. org/10.1080/10413209108406432

Eccles, J., Adler, T. F., Futterman, R., Goff, S. B., Kaczala, C. M., Meece, J. L., \& Midgley, C. (1983). Expectancies, values, and academic behaviors. En J. T. Spence (Ed.), Chievement and achievement motives (pp. 75-146). W. H. Freeman.

Eccles, J., Terry, A., \& Judith, M. (1984). Sex differences in achievement: A test of alternate theories. Journal of Personality and Social Psychology, 46(1), 26-43. https://doi.org/10.1037/00223514.46.1.26

Echavarri, M., Godoy, J., \& Olaz, F. (2007). Diferencias de género en habilidades cognitivas y rendimiento académico en estudiantes universitarios. Universitas Psychologica, 6(2), 319-329.

Frenzel, A. C., Pekrun, R., \& Goetz, T. (2007). Perceived learning environment and students' emotional experiences: A multilevel analysis of mathematics classrooms. Learning and Instruction, 17(5), 478-493. https://doi.org/10.1016/j.learninstruc.2007.09.001

Goldin, C., Katz, L. F., \& Kuziemko, I. (2006). The homecoming of american college women: The reversal of the college gender gap. The Journal of Economic Perspectives, 20(4), 133-156. https://doi.org/10.1257/jep.20.4.133

Guiso, L., Monte, F., Sapienza, P., \& Zingales, L. (2008). Culture, gender and math. Science, 320(5880), 1164-1165. https://doi. org/10.1126/science.1154094

Gunderson, E., Ramírez, G., Levine, S. C., \& Beilock, S. L. (2012). The role of parents and teachers in the development of gender-related math attitude. Sex Roles, 66(153), 153-166. https:// doi.org/10.1007/s11199-011-9996-2

Guo, J., Marsh, H. W., Morin, A. J. S., Parker, P. D., \& Kaur, G. (2015). Directionality of the associations of high school expectancy-value, aspirations, and attainment: A longitudinal study. American Educational Research Journal, 52(2), 371-402. https://doi.org/10.3102/0002831214565786

Guo, J., Marsh, H. W., Parker, P. D., \& Dicke, T. (2018). Cross-cultural generalizability of social and dimensional comparison effects on reading, math, and science self-concepts for primary school students using the combined PIRLS and TIMSS data. Learning and Instruction, 58, 210-219. https://doi.org/10.1016/j. learninstruc.2018.07.007

Harter, S. (2012). The construction of the self: A developmental perspective. Guilford.

Hartley, B. L., \& Sutton, R. M. (2013). A stereotype threat account of boys' academic underachievement. Child Development, 84(5), 1716-1733. https://doi.org/10.1111/cdev.12079 
Hyde, J. S. (2005). The gender similarities hypothesis. American Psychologist, 60(6), 581-592. https://doi.org/10.1037/0003-066X.60.6.581

Hyde, J. S. (2014). Gender similarities and differences. Annual Review of Psychology, 65, 373-398. https://doi.org/10.1146/annurev-psych-010213-115057

Jacobs, J. E., Lanza, S., Osgood, D. W., Eccles, J., \& Wigfield, A. (2002). Changes in childrens self-competence and values: Gender and domain differences across grades one through twelve. Child Development, 73(2), 509-527. https://doi.org/10.1111/14678624.00421

Kavanagh, L. (2019). Academic self-concept formation: Testing the internal/external frame of reference model, big-fish-little-pond model, and an integrated model at the end of primary school. European Journal of Psychology of Education, 35(1), 93-109. https://doi.org/10.1007/s10212-019-00416-w

Leuven, E., Oosterbeek, H., \& Van Ophem, H. (2004). Explaining international differences in male skill wage differentials by differences in demand and supply of skill. Economic Journal, 114(495), 466-486. https://doi.org/10.1111/j.1468-0297.2004.00217.x

Maccoby, E. E., \& Jacklin, C. N. (1974). The psychology of sex differences. Stanford University Press.

Marsh, H. W., \& Hau, K. (2004). Explaining paradoxical relations between academic self-concepts and achievements: Cross-cultural generalizability of the internal/external frame of reference predictions across 26 countries. Journal of Educational Psychology, 96(1), 56-67. https://doi.org/10.1037/0022-0663.96.1.56

Marsh, H. W., Pekrun, R., Murayama, K., Arens, A. K., Parker, P. D., Guo, J., \& Dicke, T. (2018). An integrated model of academic self-concept development: Academic self-concept, grades, test scores, and tracking over 6 years. Developmental Psychology, 54(2), 263-280. https://doi.org/10.1037/dev0000393

Mealey, L. (2000). Sex differences: Developmental and evolutionary strategies. Academic Press.

Meinck, S., \& Brese, F. (2019). Trends in gender gaps: Using 20 years of evidence from TIMSS. Large-Scale Assessments in Education, 7(1), 8. https://doi.org/10.1186/s40536-019-0076-3

Mullis, I. V. S., Martin, M. O., Foy, P., \& Hooper, M. (2017). PIRLS 2016 international results in reading retrieved. TIMSS \& PIRLS International Study Center.

Murnane, R. J., Willett, J. B., Yves, D., \& Tyler, J. H. (2000). How important are the cognitive skills of teenagers in predicting subsequent earnings? Journal of Policy Analysis and Management, 19(4), 547-568. https://doi.org/10.1002/1520-6688(20002 3) $19: 4<547:: A I D-P A M 2>3.0 . C O ; 2-\% 23$

OECD. (2015). ¿Qué subyace bajo la desigualdad de género en educación? PISA in focus 49. OECD Publishing.

OECD. (2016). PISA 2015 Results: Excellence and equity in education. Paris: OECD Publishing. https://doi.org/10.1787/9789264266490-en

OECD. (2018). PISA 2018: Insights and interpretations. OECD Publishing.

Paz-Albo, J., Cvencek, D., Herranz-Llácer, C. V., Hervás-Escobar, A., \& Meltzoff, A. N. (2017). Preschoolers' mathematical play and colour preferences: A new window into the development of gendered beliefs about math. Early Child Development and Care, 187(8), 1273-1283. https://doi.org/10.1080/03004430.201 7.1295234

Pinxten, M., Wouters, S., Preckel, F., Niepel, C., De Fraine, B., \& Verschueren, K. (2015). The formation of academic self-concept in elementary education: A unifying model for external and internal comparisons. Contemporary Educational Psychology, 41, 124-132. https://doi.org/10.1016/j.cedpsych.2014.12.003
Rossi, T., Trevisol, A., dos Santos-Nunes, D., Dapieve-Patias, N., \& Von Hohendorff, J. (2020). Autoeficacia general percibida y motivación para aprender en adolescentes de educación media. Acta Colombiana de Psicología, 23(1), 254-263. https://doi. org/10.14718/ACP.2020.23.1.12

Sainz, M., \& Eccles, J. (2012). Self-concept of computer and math ability: Gender implications across time and within ICT studies. Journal of Vocational Behavior, 80(2), 486-499. https://doi.org/10.1016/j.jvb.2011.08.005

Sainz, M., \& Upadyaya, K. (2016). Accuracy and bias in spanish secondary school students' self-concept of math ability: The influence of gender and parental educational level. International Journal of Educational Research, 77, 26-36. https://doi. org/10.1016/j.ijer.2016.02.009

Smith-Castro, V., Montero-Rojas, E., Moreira-Mora, T. E., \& Zamora-Araya, J. A. (2019). Expected and unexpected effects of sexism on women's mathematics performance. Interamerican Journal of Psychology, 53(1), 28-44. https://doi.org/10.30849/ rip/ijp.v53i1.905

Spencer, S. J., Steele, C. M., \& Quinn, D. M. (1999). Stereotype threat and women's math performance. Journal of Experimental Social Psychology, 35(1), 4-28. https://doi.org/10.1006/ jesp.1998.1373

Steele, C. M. (1997). A threat in the air: How stereotypes shape intellectual identity and performance. American Psychologist, 52(6), 613-629. https://doi.org/10.1037/0003-066X.52.6.613

Stoet, G., \& Geary, D. C. (2018). The gender equality paradox in science, technology, engineering, and mathematics education. Psychological Science, 29(4), 581-593. https://doi. org/10.1177/0956797617741719

Weiner, B. (1990). History of motivational research in education. Journal of Educational Psychology, 82(4), 616-622. https://doi. org/10.1037/0022-0663.82.4.616

Widlund, A., Tuominen, H., Tapola, A., \& Korhonen, J. (2020). Gendered pathways from academic performance, motivational beliefs, and school burnout to adolescents' educational and occupational aspirations. Learning and Instruction, 66(101299), 1-12. https://doi.org/10.1016/j.learninstruc.2019.101299

Wigfield, A., \& Gladstone, J.R. (2019). "What Does Expectancy-value Theory Have to Say about Motivation and Achievement in Times of Change and Uncertainty?", Motivation in Education at a Time of Global Change (pp. 15-32). https://doi.org/10.1108/ S0749-742320190000020002

Wigfield, A., \& Eccles, J. (2000). Expectancy-Value theory of achievement motivation. Contemporary Educational Psychology, 25(1), 68-81. https://doi.org/10.1006/ceps.1999.1015

Wigfield, A., Eccles, J., Fredricks, J. A., Simpkins, S., Roeser, R. W., \& Schiefele, U. (2015). Development of achievement motivation and engagement. En R. M. Lerner (Ed.), Handbook of Child Psychology and Developmental Science (7th ed.) (pp. 657-691). Wiley. https://doi.org/10.1002/9781118963418.childpsy316

Wigfield, A., Eccles, J., Yoon, K. S., Harold, R. D., Arbreton, A. J. A., Freedman-Doan, C., \& Blumenfeld, P. C. (1997). Change in children's competence beliefs and subjective task values across the elementary school years: A 3-year study. Journal of Educational Psychology, 89(3), 451-469. https://doi.org/10.1037/00220663.89.3.451 